\title{
Three-dimensional kinematic motion analysis of door handling task in people with mild and moderate stroke
}

\author{
Jung Ah Lee ${ }^{a}$, Eun Joo Kim ${ }^{b}$, Pil Woo Hwang ${ }^{c}$, Han Ram Park ${ }^{a}$, Jae Hyuk Bae ${ }^{a}$, Jae Nam Kim ${ }^{a}$ \\ ${ }^{a}$ Department of Clinical Research on Rehabilitation, National Rehabilitation Center, Seoul, Republic of Korea \\ ${ }^{b}$ Department of Physical Medicine and Rehabilitation, National Rehabilitation Center, Seoul, Republic of Korea \\ ${ }^{\mathrm{c} B a l a n c e}$ Brain Goyang Center, Goyang, Republic of Korea
}

Objective: This study aimed to quantify one of the useful upper extremity movements to evaluate motor control abilities between the groups of people with mild and moderate arm impairments performing a door handling task.

Design: Cross-sectional study.

Methods: Twenty-one healthy participants and twenty-one persons with chronic stroke ( 9 mild stroke and 12 moderate stroke) were recruited for this study. Stroke participants were divided into 2 groups based on Fugle-Meyer Assessment scores of 58-65 (mild arm) and 38-57 (moderate arm). All they performed door handling task including the pronation and supination phases 3 times. We measured some movement factors which were reaction time, movement time, hand of peak velocity, hand of movement units to perform door handling task using the three-dimensional motion analysis.

Results: The majority of kinematic variables showed significant differences among study groups $(p<0.05)$. The reaction time, total and phase of movement time, hand of peak velocity ,the number of movement units discriminated between healthy participants and persons with moderate upper limb stroke $(p<0.05)$. In addition, reaction time, total and phase of movement time, the number of movement units discriminated between those with moderate and mild upper limbs of stroke patients $(p<0.05)$.

Conclusions: Three-dimensional kinematic motion analysis in this study was a useful tool for assessing the upper extremity function in different subgroups of people with stroke during the door handling task. These kinematic variables may help clinicians understand the arm movements in door handling task and consist of discriminative therapeutic interventions for stroke patients on upper extremity rehabilitation.

Key Words: Motion analysis, Kinematic measurement, Pronation, Supination

\section{Introduction}

Stroke is a disease that causes central nervous system movement disorders and cognitive disorders, and most patients suffer from unilateral upper extremity paralysis, which contributes to movement disorders [1,2]. Upper extremity function is essential for the performance of basic activities of daily living, and because it plays an important role during other sports and leisure activities, the damaged upper extremity function of stroke patients are subjected to severe restrictions in participating in personal activities and daily life activities [3-5]. Therefore, identifying the level of restriction in daily activities due to the severity of the stroke and to accurately measure the reduced upper extremity function is necessary for the selection and application of an appropriate intervention in the area of rehabilitation [6,7].

To assess the function of the damaged upper extremity function of persons affected by stroke, the Fugl-Meyer Assessment, Frenchay Arm Test, Motor Assessment Scale, and the Action Research Arm Test was used while the Box $\&$ Block and Nine Hole Peg Tests were used as a standard measurement tool [8-10].

Received: 9 August, 2016 Revised: 2 September, 2016 Accepted: 5 September, 2016

Corresponding author: Jae Nam Kim

Department of Clinical Research on Rehabilitation, National Rehabilitation Center, 58 Samgaksan-ro, Gangbuk-gu, Seoul 01022 , Republic of Korea Tel: 82-2-901-1918 Fax: 82-2-901-1920 E-mail: nambius@korea.kr

(c) This is an Open-Access article distributed under the terms of the Creative Commons Attribution Non-Commercial License (http://creativecommons.org/licens es/by-nc/4.0) which permits unrestricted non-commercial use, distribution, and reproduction in any medium, provided the original work is properly cited.

Copyright $@ 2016$ Korean Academy of Physical Therapy Rehabilitation Science 
Although these assessment tools are considered to be reliable, they are limited in identifying small, specific changes [4]. In addition, not only is it difficult to for these tools to analyze movement patterns or the strategies used of the upper limb, it cannot exclude the subjective opinions of the examiner during the evaluation [11]. Therefore, an objective measurement tool that allows a kinematic analysis to understand the complex and sophisticated movements of the upper limb is needed $[11,12]$. The kinematic motion analysis is a method capable of measuring both quantitative and qualitative changes in motion, and is currently useful for a variety of disease groups $[13,14]$. Although motion analysis is complex, it has been reported to be effective in evaluating the characteristics of the upper extremity function of various movement disorders since it has the advantage of being able to provide the kinematic data for each joint $[15,16]$. When looking into previous studies that examine the quantitative and qualitative movements of the upper extremity through the use of kinematic motion analysis, many of them involved healthy subjects performing task-oriented reaching exercises in order to understand the mechanisms and biomechanical properties of motor control [17-19].

Although there has been several studies that have examined the ability to control upper extremity movements in persons affected by stroke, the majority of studies have focused on simple movements, such as goal-oriented reaching and pointing [13,20,21]. In addition, a study by Alt Murphy et al. [22] has reported that reaching and grasping movement abilities depended on the constraints and objectives of the task, and arm reach movements varied according to the presence or absence of a tangible object on the target point, which produces a variable kinematic movement pattern, especially during grasping or reaching an object from target point $[23,24]$. Therefore, currently there is a growing need for motion analysis studies related to task-oriented movement that can emerge during the performance of daily living activities [4]. However, studies examining upper extremity function during daily living activities in persons with stroke have only been focused on a drinking activity [22,25-28], there have been difficulties in identifying various upper extremity movements used in daily life, and relevant kinematic studies are insufficient. In order to verify the effect of intervention within persons affected by stroke, especially to differentiate the effect of moderate-level intervention on motor function, a task that is a part of daily living has to be applied and the upper extremity movement needs to be assessed for classification using the kinematic variables. Therefore, this study involved healthy subjects and subjects affected by stroke performing specific tasks of daily living (turning the door knob), and in order to investigate the function of the upper extremity kinematic differences, reaction time, movement time, peak velocity of the hand, and movement units of the hand were analyzed.

\section{Methods}

\section{Subjects}

This study included twenty-one hemiplegic subjects (16 male, 5 female) and twenty-one control subjects. The selection criteria for subjects affected by stroke were: 1) first onset of cerebral infarction or an intracerebral hemorrhage, 2) subjects with lesions confined to one side, 3) Brunnstrom stage of 3 or higher, 4) have sufficient cognitive abilities to understand and follow instructions of the investigator. Of the subjects affected by stroke, nine suffered from a mild stroke (Fugl-Meyer score, 58-65) and twelve suffered from a severe stroke (Fugl-Meyer score, 38-57). Healthy persons without any neurological and orthopedic impairments or symptoms served as the control subjects (Table 1). All subjects voluntarily provided an informed consent after being fully informed of the study purpose and methods. This study has been approved by the research ethics committee of a National Rehabilitation Center (NRC-2011-01-002).

\section{Procedure}

All subjects were instructed to sit upright on a desk and to hold onto a doorknob, which was not to be turned until at the signal of the illumination of a lamp. The doorknob turning process was composed of one continuous motion, from supination to pronation, with the ipsilateral side for subjects af-

Table 1. Characteristics of participants

$(\mathrm{N}=40)$

\begin{tabular}{lccc}
\hline \multicolumn{1}{c}{ Variable } & Healthy & $\begin{array}{c}\text { Stroke } \\
(\text { mild })\end{array}$ & $\begin{array}{c}\text { Stroke } \\
\text { (moderate) }\end{array}$ \\
\hline Sex (male/female) & $10 / 9$ & $8 / 1$ & $8 / 4$ \\
Age (y) & $23.6(3.5)$ & $42.8(18.5)$ & $53.2(14.7)$ \\
Height (cm) & $171.4(8.3)$ & $171.5(5.9)$ & $166.6(6.3)$ \\
Weight (kg) & $65.5(12.4)$ & $73.3(11.2)$ & $65.1(11.7)$ \\
Forearm length (cm) & $26.3(1.4)$ & $25.9(1.9)$ & $24.8(1.4)$ \\
Arm length (cm) & $56.2(3.4)$ & $54.7(3.2)$ & $54.0(2.6)$ \\
MMSE & - & $29.2(1.1)$ & $29.4(1.5)$ \\
Upper Fugl-Meyer & - & $61.2(3.2)$ & $49.6(6.9)$ \\
$\quad$ (score) & & & \\
\hline
\end{tabular}

Values are presented number only or mean (SD). 
fected by stroke, and with the dominant hand for the control subjects for a total of three times. To obtain data, the Vicon (Oxford, UK) eight cameras with a sampling frequency of $200 \mathrm{~Hz}$ were used. A total of 16 infrared reflective markers (14 mm marker) were applied according to the Vicon systems upper limb model mounting position. Also, an addition of five markers were attached onto the doorknob to obtain a reference point (Figure 1).
Data management and analysis

In order to analyze the behavior of the characteristics of each movement, the movement was divided into 5 subdivisions, such as reaction, supination, supination return, pronation, and pronation return (Figure 2). The reaction time, movement time, peak velocity of the hand, and movement units of the hand were analyzed according to the supination, pronation, and neutral positions. Reaction time is defined as the time it takes for trunk movement to appear at the

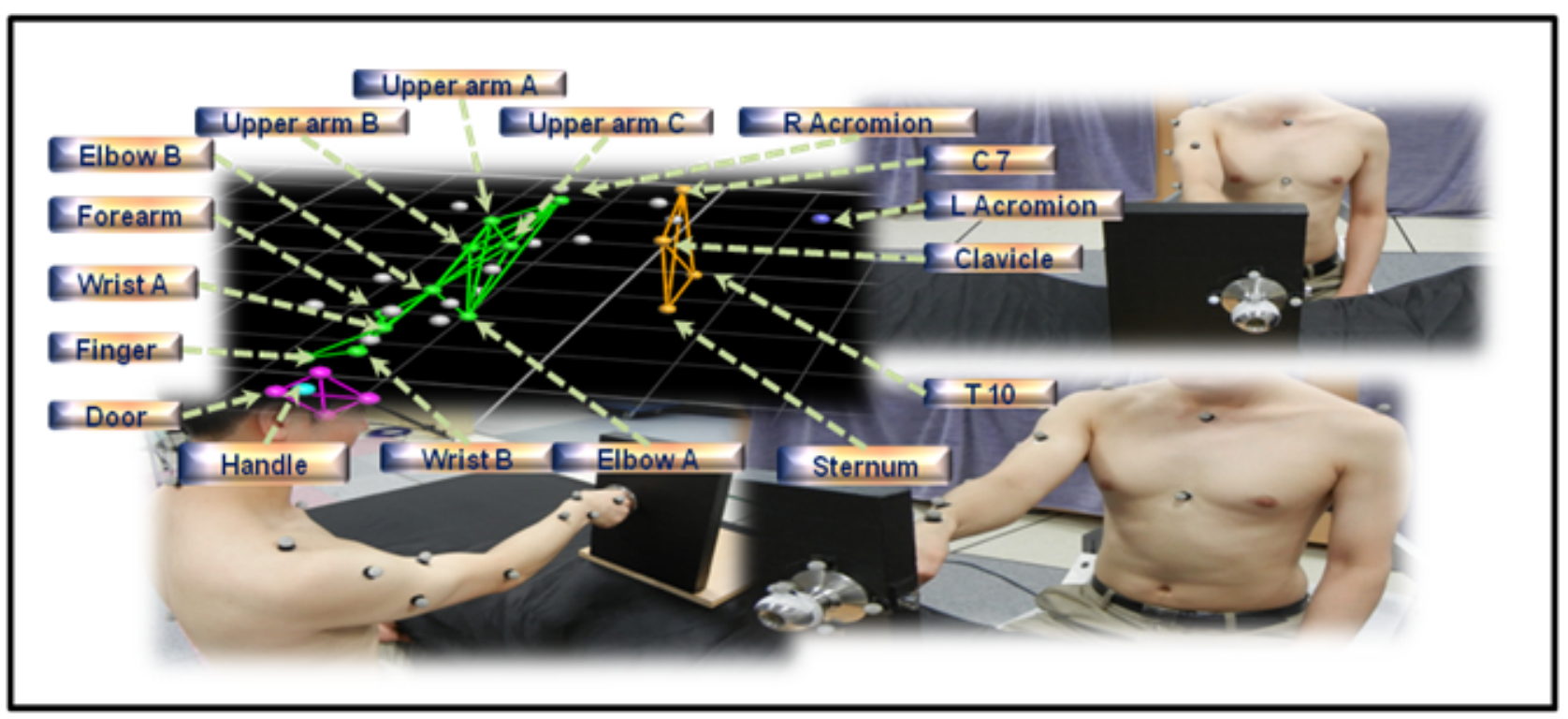

Figure 1. Marker set information.

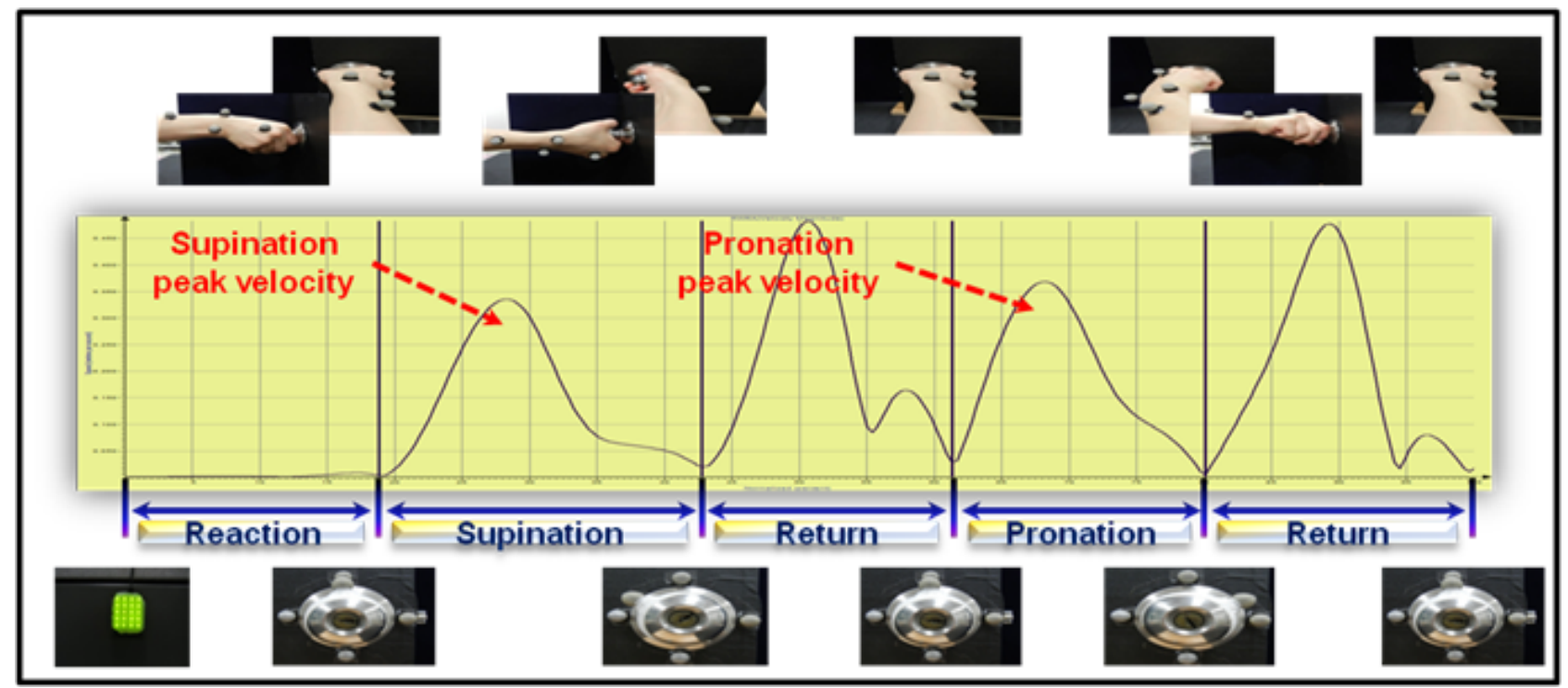

Figure 2. Phase description for door handling task. 
Table 2. Kinematic variables during door handling task

\begin{tabular}{lccrr}
\hline \multicolumn{1}{c}{ Kinematic variable } & Healthy & Stroke (mild) & Stroke (moderate) & $p$ \\
\hline Reaction time & $0.3(0.1)$ & $0.3(0.1)$ & $0.6(0.4)^{\mathrm{b}, \mathrm{c}}$ & 0.016 \\
Movement time (total) & $1.8(0.6)$ & $2.0(0.6)$ & $3.5(1.5)^{\mathrm{b}, \mathrm{c}}$ & $<0.001$ \\
Movement time (pronation phase) & $0.5(0.2)$ & $0.5(0.2)$ & $0.9(0.5)^{\mathrm{b}, \mathrm{c}}$ & 0.003 \\
Movement time (supination phase) & $0.5(0.2)$ & $0.7(0.2)^{\mathrm{a}}$ & $1.3(0.6)^{\mathrm{b}, \mathrm{c}}$ & $<0.001$ \\
Hand of peak velocity (total) & $33.8(13.4)$ & $23.1(16.7)$ & $16.8(8.6)^{\mathrm{b}}$ & 0.003 \\
Hand of peak velocity (pronation phase) & $32.5(12.4)$ & $21.4(16.3)$ & $14.9(8.4)^{\mathrm{b}}$ & 0.002 \\
Hand of peak velocity (supination phase) & $31.6(11.9)$ & $18.7(12.9)^{\mathrm{a}}$ & $13.7(8.2)^{\mathrm{b}}$ & $<0.001$ \\
Movement units (total) & $3.8(1.8)$ & $6.6(2.9)^{\mathrm{a}}$ & $13.0(7.9)^{\mathrm{b}, \mathrm{c}}$ & $<0.001$ \\
Movement units (pronation phase) & $1.8(1.0)$ & $3.0(1.6)^{\mathrm{a}}$ & $5.7(4.0)^{\mathrm{b}, \mathrm{c}}$ & $<0.001$ \\
Movement units (supination phase) & $2.0(1.1)$ & $3.6(1.5)^{\mathrm{a}}$ & $7.3(4.2)^{\mathrm{b}, \mathrm{c}}$ & $<0.001$ \\
\hline
\end{tabular}

${ }_{p}^{\mathrm{a}}<0.05$ between affected arm in participants with mild stroke and healthy participants. ${ }^{\mathrm{b}} p<0.05$ between affected arm in participants with moderate stroke and healthy participants. ${ }^{c} p<0.05$ between affected arm in participants with mild and moderate stroke.

initiation of a task, and movement time is the time it takes to perform supination and pronation.

The peak hand velocity was calculated using the tangential velocity, and the maximum velocity of each phase and the maximum velocity was measured of the total movement phase. The movement unit of the hand is defined as the acceleration and deceleration of the hand in reference to the hand markers, and the number of the movement units represents the degree of shakiness and smoothness of the hand, and the numerically smaller the movement unit of the hand, the smoother the hand movement.

When the movement unit of the hand is 1 , it represents the most optimal level of smoothness.

The unit of motion is comprised of one acceleration and one deceleration, and the numerical units within the phases of acceleration and deceleration was calculated when the motion of turning the doorknob exceeded $10 \%$ of the peak velocity of the hand movement.

The kinematic variables were analyzed using the Nexus (Vicon Inc.) software, and all data was processed using the Matlab R2012a (The MathWorks Inc, Natick, MA, USA). Statistical analysis was performed using SPSS Statistics ver. 17.0 (SPSS Inc., Chicago, IL, USA). The mean values of the general characteristics of the mild stroke, severe stroke, and control groups were used. The kinematic data was analyzed with the Mann-Whitney U test to make a comparison of the two groups. The significance level was set at $p<0.05$.

\section{Results}

Although there was no significant difference in the reaction time, total movement time between the healthy adults and mild stroke subjects, and peak velocity of the hand $(p>0.05)$, there was a significant difference in the time and velocity of the supination movement $(p<0.05)$. In addition, there was a significant difference in the movement unit of the hand during supination and pronation. There was a significant difference in reaction time, peak hand velocity, and movement units of the hand in healthy adults and mild stroke subjects $(p<0.05)$. There was a significant difference in reaction time and movement units of the hand in the mild stroke and severe stroke subjects $(p<0.05$; Table 2$)$.

\section{Discussion}

This study attempted to confirm if there was a difference in upper limb motor function in accordance to stroke severity through 3-dimenstional motion analysis during the motion of turning a doorknob, which is a common daily life activity for persons affected by stroke.

Although there was a significantly longer movement time of supination and pronation phase when comparing the healthy adults with the mild stroke group $(p<0.05)$, there was a significantly slower peak hand velocity and longer movement units of the hand comparing the mild and moderate stroke group $(p<0.05)$. In addition, there was a significantly longer in reaction time and movement units of the hand when comparing the mild with the moderate stroke group $(p<0.05)$.

In general, the movement time or strategy of a person affected by stroke is not as efficient when comparing the movement of a normal, healthy adult. Previous studies have shown that there was a large difference in the kinematic variables when not only comparing healthy adults with persons 
affected by stroke, but also when comparing between mild and severe stroke patients [22]. Therefore, it can be considered that this study has also shown a difference in the kinematic variables amongst the control group, mild stroke group, and severe stroke group.

Since this study involved task-oriented upper extremity movement, the results showed a decrease in maximum velocity of the hand, an interruption in the movement unit of the hand, leading to an overall increase in movement time, which agree with the results of previous studies [29,30]. However, a study by Alt Murphy et al. [22] compared the drinking behavior between healthy adults and persons with stroke and had found no significant difference in maximum velocity of the hand. This may be due to the fact that when persons affected by stroke move the wrist joint to turn a doorknob, there is a simultaneous contraction of the agonist and antagonist muscle groups, which is associated with spasticity, and thus, cause a delay in muscle contractions affected side [31], and also because of the reason that the muscles used during the drinking motion is different.

The motion of turning a doorknob involves supination and pronation of the forearm, where supination is performed by the forearm extensor muscles, such as the supinator muscle, and pronation is performed by the forearm flexor muscles, such as the pronator teres and pronator quadrates muscles. In regards to the Brunnstrom stages post-stroke, the higher the stage, the lower the rigidity and synergistic movements, which allows an increase in movement ease [32]. In addition, the recovery of the flexor muscles necessary to perform flexion has occurred at a lower level than the recovery of the extensor muscles to perform extension, and the flexor muscles that perform pronation have a slightly faster recovery period, indicating that it has good function. Therefore, when comparing the healthy adults with the mild stroke subjects, there was a greater significant difference in the movement time, maximum velocity of the hand and movement unit of the hand during the supination phase compared to the pronation phase during the motion of turning a doorknob.

The kinematic analysis of the distal joint while turning a doorknob can be used as an assessment tool to classify the severity of stroke for rehabilitation.

Chronic stroke patients who received twelve weeks of robotic rehabilitation training on the distal upper extremity for grasping and supination/pronation showed significant improvements in Fugl-Meyer Assessment score from 32.2 to 37.0 and Motricity Index scores from 50.6 to 57.5 [33].
The limitations of this study are as follows: 1) The age of the control and stroke subjects were not matched, 2) the flexor and extensor muscle activities were not measured. However, the fact that the kinematic variables could be quantitatively evaluated in the motor function of the wrist while turning a doorknob according to different levels of stroke severity has been shown. Therefore, in regards to upper limb function rehabilitation for patients post-stroke, discriminatory rehabilitative intervention strategies may be built and developed based upon the kinematic variables of the wrist joint during supination and pronation that have been found in this study.

\section{Conflict of Interest}

The authors declared no potential conflicts of interest with respect to the authorship and/or publication of this article.

\section{References}

1. Duncan PW, Goldstein LB, Horner RD, Landsman PB, Samsa GP, Matchar DB. Similar motor recovery of upper and lower extremities after stroke. Stroke 1994;25:1181-8.

2. Kwakkel G, Kollen BJ, Wagenaar RC. Long term effects of intensity of upper and lower limb training after stroke: a randomised trial. J Neurol Neurosurg Psychiatry 2002;72:473-9.

3. Broeks JG, Lankhorst GJ, Rumping K, Prevo AJ. The long-term outcome of arm function after stroke: results of a follow-up study. Disabil Rehabil 1999;21:357-64.

4. Alt Murphy M, Sunnerhagen KS, Johnels B, Willén C. Three-dimensional kinematic motion analysis of a daily activity drinking from a glass: a pilot study. J Neuroeng Rehabil 2006;3:18.

5. Riccio I, Iolascon G, Barillari MR, Gimigliano R, Gimigliano F. Mental practice is effective in upper limb recovery after stroke: a randomized single-blind cross-over study. Eur J Phys Rehabil Med 2010;46:19-25.

6. Caliandro P, Celletti C, Padua L, Minciotti I, Russo G, Granata G, et al. Focal muscle vibration in the treatment of upper limb spasticity: a pilot randomized controlled trial in patients with chronic stroke. Arch Phys Med Rehabil 2012;93:1656-61.

7. Tavernese E, Paoloni M, Mangone M, Mandic V, Sale P, Franceschini M, et al. Segmental muscle vibration improves reaching movement in patients with chronic stroke. A randomized controlled trial. NeuroRehabilitation 2013;32:591-9.

8. Finch E, Brooks D, Stratford PW, Mayo NE. Physical rehabilitation outcome measures: a guide to enhanced clinical decision making. 2nd ed. Hamilton (ON): BC Decker; 2002.

9. McCrea PH, Eng JJ, Hodgson AJ. Biomechanics of reaching: clinical implications for individuals with acquired brain injury. Disabil Rehabil 2002;24:534-41.

10. Wade DT. Measurement in neurological rehabilitation. Curr Opin Neurol Neurosurg 1992;5:682-6. 
11. de los Reyes-Guzmán A, Dimbwadyo-Terrer I, Trincado-Alonso F, Monasterio-Huelin F, Torricelli D, Gil-Agudo A. Quantitative assessment based on kinematic measures of functional impairments during upper extremity movements: a review. Clin Biomech (Bristol, Avon) 2014;29:719-27.

12. Cacho EW, de Oliveira R, Ortolan RL, Varoto R, Cliquet A Jr. Upper limb assessment in tetraplegia: clinical, functional and kinematic correlations. Int J Rehabil Res 2011;34:65-72.

13. Kamper DG, McKenna-Cole AN, Kahn LE, Reinkensmeyer DJ. Alterations in reaching after stroke and their relation to movement direction and impairment severity. Arch Phys Med Rehabil 2002;83:702-7.

14. Trombly CA. Deficits of reaching in subjects with left hemiparesis: a pilot study. Am J Occup Ther 1992;46:887-97.

15. Gentilucci M, Benuzzi F, Gangitano M, Grimaldi S. Grasp with hand and mouth: a kinematic study on healthy subjects. J Neurophysiol 2001;86:1685-99.

16. Paulignan Y, MacKenzie C, Marteniuk R, Jeannerod M. The coupling of arm and finger movements during prehension. Exp Brain Res 1990;79:431-5.

17. Maitra KK, Junkins MD. Upper extremity movement pattern of a common drinking task in well elderly women: a pilot study. Occup Ther Int 2004;11:67-81.

18. Murgia A, Kyberd PJ, Chappell PH, Light CM. Marker placement to describe the wrist movements during activities of daily living in cyclical tasks. Clin Biomech (Bristol, Avon) 2004;19: 248-54.

19. Weiss PH, Jeannerod M, Paulignan Y, Freund HJ. Is the organisation of goal-directed action modality specific? A common temporal structure. Neuropsychologia 2000;38:1136-47.

20. Cirstea MC, Mitnitski AB, Feldman AG, Levin MF. Interjoint coordination dynamics during reaching in stroke. Exp Brain Res 2003;151:289-300.

21. Levin MF. Interjoint coordination during pointing movements is disrupted in spastic hemiparesis. Brain 1996;119:281-93.

22. Alt Murphy M, Willén C, Sunnerhagen KS. Kinematic variables quantifying upper-extremity performance after stroke during reaching and drinking from a glass. Neurorehabil Neural Repair 2011;25:71-80.

23. Shumway-Cook A, Woollacott MH. Motor control: theory and practical applications. Baltimore: Lippincott Williams \& Wilkins; 1995.

24. Trombly CA, Wu CY. Effect of rehabilitation tasks on organization of movement after stroke. Am J Occup Ther 1999;53: 333-44.

25. Aprile I, Rabuffetti M, Padua L, Di Sipio E, Simbolotti C, Ferrarin M. Kinematic analysis of the upper limb motor strategies in stroke patients as a tool towards advanced neurorehabilitation strategies: a preliminary study. Biomed Res Int 2014; 2014:636123.

26. Kim K, Song WK, Lee J, Lee HY, Park DS, Ko BW, et al. Kinematic analysis of upper extremity movement during drinking in hemiplegic subjects. Clin Biomech (Bristol, Avon) 2014; 29:248-56.

27. Alt Murphy M, Willén C, Sunnerhagen KS. Movement kinematics during a drinking task are associated with the activity capacity level after stroke. Neurorehabil Neural Repair 2012;26: 1106-15.

28. Wu CY, Wong MK, Lin KC, Chen HC. Effects of task goal and personal preference on seated reaching kinematics after stroke. Stroke 2001;32:70-6.

29. Lough S, Wing AM, Fraser C, Jenner JR. Measurement of recovery of function in the hemiparetic upper limb following stroke: a preliminary report. Human Mov Sci 1984:3:247-56.

30. Trombly CA. Observations of improvement of reaching in five subjects with left hemiparesis. J Neurol Neurosurg Psychiatry 1993;56:40-5.

31. Chae J, Yang G, Park BK, Labatia I. Delay in initiation and termination of muscle contraction, motor impairment, and physical disability in upper limb hemiparesis. Muscle Nerve 2002;25: 568-75.

32. De Weerdt WJG, Harrison MA. Measuring recovery of arm-hand function in stroke patients: a comparison of the BrunnstromFugl-Meyer test and the action research arm test. Physiother Canada 1985:37:65-70.

33. Lambercy O, Dovat L, Yun H, Wee SK, Kuah CW, Chua KS, et al. Effects of a robot-assisted training of grasp and pronation/ supination in chronic stroke: a pilot study. J Neuroeng Rehabil 2011;8:63. 